\title{
Human Lactoferrin Peptide hLF1-11
}

National Cancer Institute

\section{Source}

National Cancer Institute. Human Lactoferrin Peptide hLF1-11. NCI Thesaurus. Code C67046.

A synthetic peptide corresponding to the first $11 \mathrm{~N}$-terminal amino acids of human lactoferrin (hLF1-11) with potential antimicrobial activity. Human lactoferrin, a 692 amino acid glycoprotein belong ing to the transferrin family of metal-binding proteins, can be found in milk and other secretory fluids as well as in polymorphonuclear cells and leukocytes. Human lactoferrin plays a role in the innate defense of mucosal surfaces and neutrophils. Although the exact mechanism through which this peptide exerts its effect has yet to be fully elucidated, hLF1-11 may seize iron, thereby limiting the availability of free iron for microorganisms in the body. hLF1-11 appears to be effective against a variety of bacteria and fungi, including the multidrug-resistant Acinetobacter baumannii, multidrug-resistant Staphylococcus aureus, and fluconazole-resistant Candida albicans strains. 